\title{
Limited evidence for existing caries assessment systems
}

\section{Abstracted from}

Tellez M, Gomez J, Pretty I, Ellwood R, Ismail A.

Evidence on existing caries risk assessment systems: are they predictive of future caries? Community Dent Oral Epidemiol 2012; Sep 15. doi: 10.1111/cdoe.12003.

[Epub ahead of print] PubMed PMID: 22978796.

Address for correspondence: Marisol Tellez, Maurice H Kornberg School of Dentistry, Temple University, Philadelphia, PA, USA. E-mail: marisol@dental.temple.edu

\section{Question: Are current caries risk assessment systems predictive of future caries?}

Data sources Data were sourced from the Cochrane Oral Health Groups Specialised Register, Cochrane Central Register of Controlled Trails, Medline, bibliographic references of identified systematic reviews, prospective cohort studies and clinical trials, textbooks and review articles.

Study selection The studies included presented validating criteria for caries incidence/ increment and were limited to those with human subjects and natural carious lesions. Only studies published in peer reviewed journals were included. Excluded were studies which gave an incomplete description of sample selection, or of outcome, or had a small sample size. Studies which did not meet the Oxford Centre for Evidence Based Medicine prognosis category criteria for best evidence were also excluded. Data extraction and synthesis Data were extracted by the first review author and were independently checked by a second author. The criteria reported in the ADA Clinical Recommendations Handbook ${ }^{1}$ were used to assess the quality of the studies. Adjustments made for potential confounders were considered as a means to evaluate the internal validity of each study.

Results One hundred and thirty-seven study reports remained for review following systematic strategic search and title review. Of these, six studies of existing caries risk assessment models were selected for inclusion. Of the six studies reviewed four were deemed 'fair' by the ADA criteria and two 'poor'. The authors found variation in the parameters used for caries risk assessment and the population groups studied. No study found the risk assessment systems to have reliable prediction utility in children. One prospective study found Cariogram to give good to moderate caries prediction in elderly adults and one retrospective study found the CAMBRA assessment to provide prediction for cavitated lesions, but only between low risk and extreme risk individuals over the age of six.

Conclusions This systematic review suggests that evidence available on the validity of a number of existing systems for caries risk assessment is limited and weak.

\section{Commentary}

The identification of high risk individuals to allow both prevention and intervention based on susceptibility to disease is commonplace in contemporary treatment planning. A systematic review of the literature by Harris et al in 2004 found ' 106 risk factors significantly related to the prevalence or incidence of caries' 2 . In general caries risk assessment systems standardise the risk factor information collected in order to predict potential caries outcome for the patient.

This systematic review examines the evidence on whether existing caries risk assessment systems are predictive of future caries. In addition, a second research question: 'What are the outcomes of management based on the use of these systems?' is presented within the introduction to the review. The authors searched three relevant databases and appropriate reference lists. The search was restricted to articles where one of four specific caries risk assessment models was used. It is possible that additional caries risk assessment models such as the recently published Dundee Caries Risk Assessment (DCRAM)3 may have been found by including further databases and, or extending the search to include unpublished literature. No restrictions were put on the population group to be studied in terms of age or stage of dental development. Limited evidence to answer either of the proposed research questions was found.

The inclusion of prospective and retrospective cohort studies allowed the predictive capability for of each of the caries risk assessments to be assessed in terms of an increase in the clinical caries incidence over time. Both cohort and randomised control trial studies which met the inclusion criteria were included for review. Randomised control trials would generally be included within a review where a specific intervention is being tested. Only one randomised control trial which met the inclusion criteria was included. This significantly limited the evidence available to review with regard to the second additional research question.

Six studies were reviewed by the authors. A narrative review of each individual study is provided alongside a table of result characteristics. Summary statistics from each study were described and discussed. Meta-analysis was not carried out for this review. The authors found variation in the parameters used for caries risk assessment and the population groups studied. Published evidence was found for only two of the four selected caries risk assessment systems. The same caries risk assessment program was used in five of the six articles which met the search criteria. These five studies were all carried out in Sweden, with three performed on the same 
sample of children. A quality assessment was performed and found two of the six studies of poor quality, the remaining four studies were deemed to be fair. One study included in the review looked at caries risk assessment in an adult population. The results of this describe good to moderate caries prediction for extreme risk adult subjects but should be interpreted with caution given the quality of the study. Eight predictive models in longitudinal studies were also tabled for discussion within the article but were not reviewed in full.

The authors of this article have provided a structured review. There are limitations with regard to the quality of the evidence retrieved and the content of the review. Nevertheless, the authors bring to the attention of the dental research community the difficulties and limitations of caries risk assessment.
This is an area which warrants research development and further systematic review.

\section{Susan J Carson}

Dundee Dental School, Park Place, Dundee, Scotland, UK

1. Center for Evidence Based Dentistry American Dental Association. ADA Clinical Recommendations Handbook. October 29, 2009.

2. Harris R, Nicoll AD, Adair PM, Pine CM. Risk factors for dental caries in young children: a systematic review of the literature. Community Dental Health 2004. 21 (Suppliment), 71-85.

3. MacRitchie HM, Longbottom C, Robertson M, Nugent Z, Chan K, Radford JR, Pitts NB. Development of the Dundee Caries Risk Assessment Model (DCRAM) - risk model development using a novel application of CHAID analysis. Community Dentistry and Oral Epidemiology 2012. Feb; 40: 37-45

Evidence-Based Dentistry (2013) 14, 10-11. doi:10.1038/sj.ebd.6400911 